\title{
Síntesis de nanopartículas de magnetita a partir del extracto de cáscara de papaya para la degradación de colorantes azoicos en soluciones acuosas
}

\author{
Synthesis of magnetite nanoparticles using papaya peel extract \\ for the azo dyes degradation in aqueous solutions
}

\author{
Diana Paola Robles Ardila ${ }^{1} \quad$ Natalia Rodríguez Pardo ${ }^{1} \quad$ Alis Pataquiva-Mateus ${ }^{1 *}$ \\ Recibido 17 de marzo de 2017, aceptado 18 de julio de 2018 \\ Received: March 17, 2017 Accepted: July 18, 2018
}

\begin{abstract}
RESUMEN
El presente documento describe el proceso realizado para sintetizar nanopartículas de magnetita $\mathrm{Fe}_{3} \mathrm{O}_{4}$ a partir de extracto de cáscara de papaya mediante una reacción simple y ecológica. Para la realización de la síntesis se verifica la cantidad de glucosa presente en las cáscaras puesto que este carbohidrato es indispensable como agente reductor en la reacción, el procedimiento se realiza para tres muestras diferentes las cuales son evaluadas por medio de microscopia infrarroja FTIR y comparadas con una muestra comercial, por otro lado, se evidencia por medio de una prueba SEM si el tamaño de los productos obtenidos corresponden a material nanométrico y si la morfología efectivamente corresponde a magnetita; posterior a esto se realiza la degradación de colorantes azoicos dando resultados positivos para el colorante azul brillante E-133 mostrando transparencia en el medio lo que se verifica de manera cuantitativa por medio de espectroscopia UV-Vis.
\end{abstract}

Palabras clave: Magnetita, papaya, colorantes azoicos, nanopartículas.

\begin{abstract}
This document describes the $\mathrm{Fe}_{3} \mathrm{O}_{4}$ magnetite nanoparticles process synthesis via papaya peel extract by a simple and ecological reaction. For the synthesis, is verified the amount of glucose present in the shells since this carbohydrate is indispensable as a reducing agent in the reaction, the method is make for three different samples that are evaluated by infrared microscopy FTIR and compared with a commercial sample, in the other hand by SEM was possible see if the size and morphology corresponding to the expected results; subsequent the azo dyes degradation give positive results for the bright blue E-133 dye showing the transparency in the medium and is checked quantitatively through of UV-Vis spectroscopy.
\end{abstract}

Keywords: Magnetite, papaya, azo dyes, nanoparticles.

\section{INTRODUCCIÓN}

Uno de los grandes problemas ambientales que se da en los tiempos actuales está relacionado con el uso desmesurado del recurso hídrico, ya que este es de vital importancia para todos los procesos productivos; sin embargo, en algunos casos no es necesario que se haga uso de agua potable, por tal motivo es indispensable conocer nuevos métodos que además de ser ecológicos permitan la remoción de diferentes tipos de contaminantes, reduciendo así el gasto de agua y los costos de operación [1].

\footnotetext{
1 Universidad Jorge Tadeo Lozano. Departamento de Ingeniería. Carrera 4 22-61. Bogotá, Colombia.

E-mail: dianap.roblesa@utadeo.edu.co; natalia.rodriguezp@utadeo.edu.co; alisy.pataquivam@utadeo.edu.co

* Autor de correspondencia: alisy.pataquivam@utadeo.edu.c
} 
Los colorantes azoicos disueltos en agua derivan un serio problema medioambiental puesto que no pueden ser tratados en una planta de tratamiento de aguas y además afectan los procesos acuáticos debido a que impiden el paso de la luz a través del agua [2].

Otro problema ambiental importante se da con la generación de residuos agroindustriales, es decir materiales bien sea sólidos o líquidos generados a partir del consumo de una materia prima y que contienen cierto potencial para ser usados en otros procesos, dar un mejor aprovechamiento o transformación en un producto comercial, así, pues, este tipo de residuos pueden conllevarles a las industrias un ingreso adicional y la disminución de los gastos de disposición de los mismos [3].

Basado en lo anterior y conociendo que Colombia es un país ubicado en el trópico es posible encontrar zonas en donde se generan este tipo de residuos a partir de diferentes fuentes, por ejemplo, la papaya, fruto del cual sus residuos son la base de la presente investigación.

La papaya debido a su alto valor nutritivo y propiedades medicinales es un fruto que ha logrado incrementar su cultivo, haciendo que se comercialice no solo en las regiones de América (de donde es nativa) sino también en África, Asia, Australia, Filipinas y Estados unidos de América, es una fruta con forma ovalada y un peso promedio de $500 \mathrm{~g}$, el árbol puede crecer en casi cualquier tipo de suelo que sea ligero, profundo, fértil y permeable, aunque se da mejor en climas cálidos o templados [4].

La cáscara de papaya es un residuo agroindustrial potencial debido a los diferentes componentes presentes en ella tales como fibra insoluble $26 \%$, proteínas $18 \%$, carbohidratos $9.69 \%$, lípidos $2,4 \%$ y ceniza $11,85 \%$ entre otros [5].

De los cuales serán de interés los carbohidratos por su comportamiento como agente reductor frente a la hematita $\left(\mathrm{Fe}_{2} \mathrm{O}_{3}\right)$.

La producción anual de papaya en Colombia según estudios reportados hasta el 2010, es de $30 \mathrm{mil}$ toneladas al año y equivale al 10,20\% de las frutas que produce el país [6]; se sabe que el exocarpio (cáscara de la fruta) comprende entre un 17 - 20\% de la fruta aproximadamente lo que equivaldría a
6000 toneladas al año [7]. Por ejemplo, en Colombia en ciudades como Medellín y el Valle del Aburrá se llega a producir $100 \mathrm{~kg} /$ día de cáscara de papaya [8].

Las nanopartículas (NPs) se han convertido en uno de los objetivos principales de la ciencia, ya que a esta escala es posible obtener propiedades de los compuestos que a mayor escala no se podrían aprovechar, dentro de estas las nanopartículas metálicas han atraído el interés durante las últimas dos décadas debido a sus propiedades optoelectrónicas y fisicoquímicas [9]; también se ha evaluado la posibilidad de aprovechar sus propiedades magnéticas en pro de la salud humana [10].

Existe una variedad de métodos para llevar a cabo la síntesis de estas nanopartículas, entre ellos, el método sonoquímico, microemulsiones, electroquímico y de coprecipitación, cada uno de estos usa compuestos en cuya estructura hay presencia de nitrógeno tales como urea, amoníaco, etc. generando afecciones ambientales [11].

Por tal motivo se empezó a estudiar un método, que además de evitar el uso de dichos compuestos, aprovecha residuos agroindustriales tales como la cáscara del banano y la sandía conociendo que en estas hay azúcares reductores presentes. Con el fin de concentrar los azúcares, las cáscaras fueron sometidas a un proceso de reflujo a diferentes condiciones, luego de haber removido la humedad, estas se pulverizaron y se disolvieron en cloruro de hierro hexahidratado y acetato de sodio, esta mezcla fue llevada a calentamiento para la posterior obtención de las NPs [12].

La magnetita de tamaño nanométrico comprende amplias aplicaciones en biodetección, el uso futuro en tratamientos contra el cáncer [13], la catálisis y remediación ambiental [9], siendo en esta última donde las NPs se han convertido en adsorbentes de contaminantes presentes en el agua, ya que las características de estos materiales están relacionadas con la capacidad para eliminar iones de metales pesados [14] y diferentes tipos de colorantes, entre los cuales se encuentran los colorantes azoicos [15].

\section{ANÁLISIS TEÓRICO}

Teniendo en cuenta las propiedades magnéticas que tiene la magnetita, ésta se ha obtenido por medio de la 
síntesis de expansión reductiva (RES), la cual se basa en la reacción que se da entre la urea que debe estar en exceso y compuestos de nitrato en una atmósfera inerte y en calentamiento a alta temperatura con el fin de obtener partículas de metales con valencia 0 como primer producto, con el transcurrir del tiempo y otro calentamiento rápido se pueden obtener las NPs de interés. El análisis consistió en observar el comportamiento de la reacción bajo una atmósfera oxidante, determinándose por difracción de rayos $\mathrm{x}$ que la reacción descrita anteriormente bajo esta condición sí sintetiza las partículas esperadas; sin embargo, el diámetro de las mismas superó los $100 \mathrm{~nm}$ indicando que son submicrométricas [16].

Con el fin de determinar la posibilidad de obtener NPs de magnetita por medio de un método de síntesis verde, se estudió si el uso de un azúcar reductor permitiría llevar a cabo la síntesis, para esto se usó como fuente la cáscara de banano, residuo escogido teniendo en cuenta su alta generación y la poca cantidad de estudios que se han hecho respecto a los usos que se le podría dar a partir de su composición química; lo que se buscaba era determinar si la capacidad reductora de glucosa presente en la cáscara era útil para la generación del producto de interés (ecuaciones (1-3)), para esto la glucosa fue concentrada en un extracto obtenido de las cáscaras.

$$
\begin{gathered}
2 \mathrm{Fe}^{3+}+\mathrm{CH}_{3} \mathrm{COO}^{-}+5 \mathrm{H}_{2} \mathrm{O} \vec{\Delta} \mathrm{Fe}_{2} \mathrm{O} \\
\left(\mathrm{CH}_{3} \mathrm{COO}\right)(\mathrm{OH})_{3} \cdot \mathrm{H}_{2} \mathrm{O}+5 \mathrm{H}^{+} \\
\mathrm{Fe}_{2} \mathrm{O}\left(\mathrm{CH}_{3} \mathrm{COO}\right)(\mathrm{OH}) \cdot \mathrm{H}_{2} \mathrm{O} \vec{\Delta} \\
\mathrm{Fe}_{2} \mathrm{O}_{3}+\mathrm{CH}_{3} \mathrm{COOH}+2 \mathrm{H}_{2} \mathrm{O} \\
3 \mathrm{Fe}_{2} \mathrm{O}_{3}+\mathrm{C}_{6} \mathrm{H}_{12} \mathrm{O}_{6} \vec{\Delta} \\
2 \mathrm{Fe}_{3} \mathrm{O}_{4}+\mathrm{C}_{6} \mathrm{H}_{12} \mathrm{O}_{7}
\end{gathered}
$$

Se han desarrollado investigaciones con el fin de determinar el efecto catalítico de la magnetita acompañada de otros compuestos en la reducción de colorantes azoicos que estén presentes en el agua [17], el ensayo consistió en sintetizar un catalizador cuya estructura es $\mathrm{Fe}_{3} \mathrm{O}_{4}+\mathrm{NiCo}+\mathrm{Cu}$ (Magnetita, ácido nicotínico introducido, y cobre) a partir de sales metálicas disueltas en agua destilada con ácido nicotínico, una solución concentrada de amoníaco, y una solución de $\mathrm{Cu}\left(\mathrm{NO}_{3}\right)_{2}$, luego de realizar los correspondientes análisis, se encontró que el tipo de nanocatalizadores sintetizados permiten la degradación química y biológica de los colorantes azoicos lo que evidencian por medio de pruebas de UV-Vis [9].

Derivado de los anteriores estudios se determinó la siguiente metodología para la síntesis verde de nanopartículas de $\mathrm{Fe}_{3} \mathrm{O}_{4}$ a partir del extracto de la cáscara de papaya.

\section{SECCIÓN EXPERIMENTAL}

\section{Preparación del extracto de la cáscara de papaya}

La cáscara recolectada fue lavada con agua destilada y dividida en dos grupos, uno en el cual la cáscara se encontraba más fresca (A) y otra que se encontraba con más días de almacenamiento (B) con una humedad inicial de $97,57 \%$ en un tiempo de $13: 58$ min y $73,84 \%$ en 11:17 min respectivamente, evidenciando así que el primer grupo comprendía una mayor humedad.

Posteriormente, ambos grupos de cáscara fueron sometidos a un proceso de secado a una temperatura de $60{ }^{\circ} \mathrm{C}$ por 24 horas, la cáscara seca fue cortada en trozos de aproximadamente $2 \times 2 \mathrm{~cm}$ y al medir la humedad final se obtuvieron valores de $22,07 \%$ en un tiempo de 5 min y de $23,15 \%$ en 8 min para las cáscaras A y B, respectivamente.

Para ambos grupos de cáscara se realizó un montaje soxhlet a una relación 1/10 cáscara/agua con $10 \mathrm{~g}$ de cada cáscara en $100 \mathrm{~mL}$ de agua destilada, este montaje se realizó con el fin de concentrar la glucosa presente en la cáscara [16] y se llevó a cabo por una hora en un rango de temperatura entre 70 y $80{ }^{\circ} \mathrm{C}$ con agitación magnética constante. Finalmente, los extractos obtenidos de ambos grupos, los cuales se denominarán: Extracto 1 (E1), para el extracto con una humedad final de $22,07 \%$ (cáscara A) y Extracto 2 (E2) para el que obtuvo una humedad final de $23,15 \%$ (cáscara B); fueron llevados a refrigeración por 24 horas.

\section{Determinación de glucosa}

Con el fin de determinar la presencia de azúcares reductores en el extracto que permitan llevar a cabo la reacción presentada en la ecuación (3), se empleó el método espectrofotométrico con el uso de DNS para establecer la cantidad exacta de carbohidratos presentes como glucosa [18]. 
Se tomó una curva patrón realizada a partir de una solución de glucosa de concentración $2 \mathrm{~g} / \mathrm{L}$, y con diluciones de las muestras de extracto a analizar.

\section{Síntesis de nanopartículas de $\mathrm{Fe}_{3} \mathrm{O}_{4}$}

Teniendo en cuenta las ecuaciones (1), (2) y (3) se agregaronn diferentes excesos de acetato de sodio a una cantidad inicial de cloruro de hierro hexahidratado $\mathrm{FeCl}_{3} \cdot \mathrm{H}_{2} \mathrm{O}$, se someten a calentamiento $\left(60^{\circ} \mathrm{C}\right)$ hasta la formación de un compuesto de color rojo ladrillo característico de la formación del óxido de hierro II o hematita el cual es el producto en la ecuación (2). Posteriormente, se agregaron $20 \mathrm{~mL}$ del extracto de glucosa extraído anteriormente y, manteniendo una temperatura de $60^{\circ} \mathrm{C}$. Finalmente, las muestras fueron llevadas a la mufla a una temperatura de $500^{\circ} \mathrm{C}$ por 24 horas eliminándose así el ácido acético formado, con el fin de favorecer la formación del compuesto metálico de interés.

\section{Caracterización}

\section{- Química}

Con el fin de verificar que el compuesto obtenido es magnetita, se llevó a cabo la espectrometría infrarroja con transformada de Fourier (FTIR, de sus siglas en inglés) en el equipo Cary 630 FTIR con el fin de determinar los grupos funcionales presentes en el compuesto resultante. Para llevar a cabo el análisis fue necesario pulverizar la muestra hasta un tamaño inferior al de la longitud de onda de la radiación para evitar los efectos de dispersión debidos a la misma [19].

\section{- Física}

El compuesto obtenido fue analizado por medio de microscopía electrónica de barrido (SEM, de sus siglas en inglés) para comprobar que tiene un tamaño nanométrico y su morfología corresponde a la característica de las nanopartículas de magnetita. El análisis SEM fue llevado a cabo en el microscopio JEOL JSM-6490LV. La muestra fue esparcida sobre etanol y llevada a ultrasonido, consecutivamente se tomó una gota de la suspensión formada y se colocó sobre un pin previamente calentado, el etanol ayuda a que la muestra se esparza uniformemente sobre el pin. La muestra fue recubierta con oro usando el metalizador Desk $®$ IV para su posterior visualización.

Teniendo en cuenta que el compuesto sintetizado tiene como característica el magnetismo, este se sometió a un campo magnético con el fin de verificar que tuviera dicha propiedad.

\section{- Determinación de la remoción de colorantes} Con el propósito de determinar si las NPs de magnetita sintetizadas tienen un efecto de remoción frente a colorantes azoicos presentes en el agua, se evaluó la degradación del colorante Azul Brillante $(\mathrm{AB})$ a través del tiempo por medio del método de espectroscopia UV-Vis en el equipo Evolution 300 a una longitud de onda de $555 \mathrm{~nm}$ [20].

El instrumento mide la potencia del haz de luz que pasa a través de la solución del analito y la compara con la potencia del haz emitido por una muestra que contiene solo el solvente, la concentración de un analito absorbente se relaciona en forma lineal con la absorbancia según la ley de Beer [21], según se indica en ecuación (4):

$$
A=-\log T=\log P 0 P=\varepsilon b c
$$

Donde:

$A=\log P_{0} / P=$ Absorbancia

$T=P / P_{0}=$ Transmitancia

$\varepsilon=$ Absortividad molar

$b=$ Longitud de la celda en $\mathrm{cm}$

$c=$ Concentración del analito

Las diferentes concentraciones del analito en cuestión (colorante) fueron determinadas por medio de una curva de calibración realizada con concentraciones conocidas del analito y la cual, de acuerdo con su linealidad, brindaron la relación entre absorbancias y concentraciones de este con la ecuación de la recta.

\section{RESULTADOS Y DISCUSIÓN}

\section{Cuantificación de glucosa}

Se llevó a cabo la preparación de los extractos E1 y E2, los cuales fueron analizados a dos diferentes volúmenes como se indica en la Tabla 1 para determinar su concentración por medio de la técnica de Miller, datos consignados en la Figura 1, en esta se muestra la curva de glucosa leída en el espectro UV-Vis con el método fijo a una longitud de onda de $540 \mathrm{~nm}$, con ayuda de la linealidad de la curva de la glucosa se determinó la ecuación de la recta a partir de la cual se calculó la concentración de glucosa de cada muestra resultante de los reflujos, datos consignados en la Tabla 1. 
Tabla 1. Volúmenes, absorbancia y concentración inicial y final de las muestras problema.

\begin{tabular}{|c|c|c|c|c|c|c|}
\hline \multicolumn{2}{|c|}{ Muestra } & $\begin{array}{c}\text { Volumen Muestra } \\
(\mathbf{m l})\end{array}$ & $\begin{array}{c}\text { Volumen Agua } \\
(\mathbf{m l})\end{array}$ & ABS & $\begin{array}{c}\text { Concentración } \\
{[\mathbf{m g} / \mathrm{L}]}\end{array}$ & \multirow{2}{*}{ Promedio } \\
\hline \multirow{2}{*}{ E1 } & a & 0,2 & 4,2 & 0,280 & 25,304 & \multirow{2}{*}{28,148} \\
\cline { 2 - 7 } & b & 0,4 & 4,0 & 0,685 & 30,991 & \multirow{2}{*}{ E2 } \\
\cline { 2 - 6 } & c & 0,2 & 4,2 & 0,013 & 23,020 & 19,780 \\
\hline
\end{tabular}

\section{Cuantificación de glucosa}

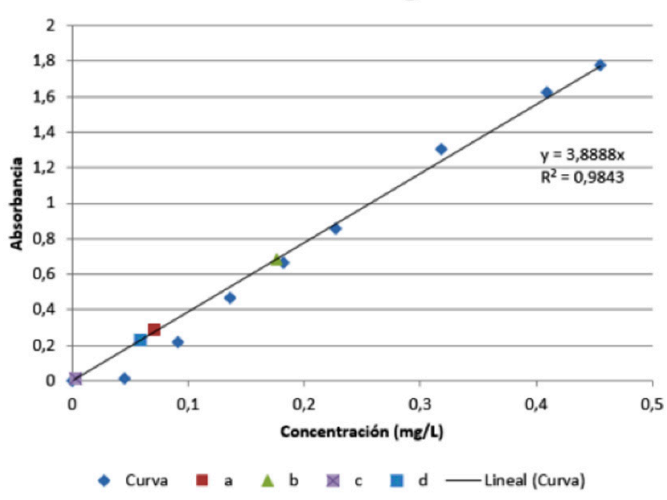

Figura 1. Cuantificación de glucosa por medio del método fijo por espectroscopía UV-Vis.

\section{Excesos}

Con el fin de determinar la posibilidad de sintetizar magnetita variando las cantidades de reactivos reportados, se optó por añadir diferentes excesos de acetato de sodio a una cantidad cercana a $2.16 \mathrm{~g}$ de $\mathrm{FeCl}_{3} \cdot \mathrm{H}_{2} \mathrm{O}$ como se muestra en la Tabla 2. Posteriormente, se determinó la masa de magnetita obtenida para cada exceso (Tabla 3).

A partir de lo anterior, se determinó que sí es posible la obtención de magnetita disminuyendo el exceso de 20:1 a 10:1, lo cual se puede identificar en la

Tabla 2. Proporción de reactivos y cantidad de exceso molar de acetato de sodio $\left(\mathrm{C}_{2} \mathrm{H}_{3} \mathrm{NaO}_{2}\right)$ con respecto al cloruro de hierro $\left(\mathrm{FeCl}_{3}\right)$.

\begin{tabular}{|c|c|c|c|c|}
\hline \multicolumn{2}{|l|}{ Muestra } & $\begin{array}{c}\text { Masa } \mathrm{FeCl}_{3} \\
(\mathbf{g})\end{array}$ & $\begin{array}{c}\mathrm{Masa}_{\mathbf{2}} \mathbf{C}_{\mathbf{3}} \mathbf{N a O}_{\mathbf{2}} \\
(\mathbf{g})\end{array}$ & Excesos \\
\hline \multirow{2}{*}{ E1 } & M1 & 2,079 & 3,252 & 10 \\
\cline { 2 - 5 } & M2 & 2,271 & 4,893 & 15 \\
\hline E2 & M3 & 2,170 & 6,524 & 20 \\
\hline
\end{tabular}

Figura 2 ya que en el espectro obtenido se observa que los picos característicos de la zona perteneciente a enlaces Fe-O guardan similitudes con respecto al pico de la muestra comercial, como por ejemplo el ancho del mismo, de lo anterior es posible afirmar que se disminuye la generación de ácido acético en un $50 \%$ con respecto a la muestra en la que se agregó un exceso de aproximadamente 20:1, exceso que ha sido reportado previamente por diferentes investigaciones como el uso de extractos obtenidos a partir de cáscaras de banano [14], sandía [12] y extracto de semilla de Syzygium cumini [22].

Teniendo en cuenta lo anterior se puede deducir entonces que habrá una mayor cantidad de magnetita obtenida en el exceso 10:1 que en el exceso 20:1, ya que la glucosa tiene un mayor número de moles de hematita con las que podrá reaccionar reduciéndolas a magnetita.

\section{Rendimientos}

La determinación del porcentaje de rendimiento se basa en los coeficientes estequiométricos presentados en las ecuaciones (1-3) [23] y los excesos presentados

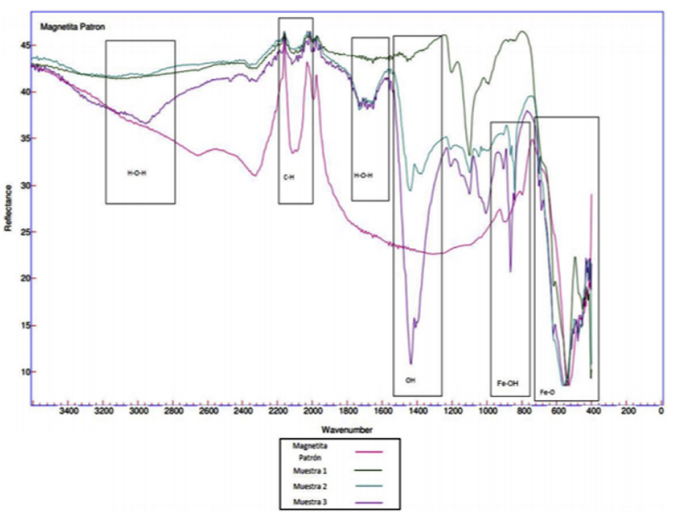

Figura 2. Comparación de espectros de las muestras con magnetita comercial. 
en la Tabla 2. Para este caso se analiza el rendimiento de la magnetita con respecto a la cantidad de cloruro de hierro usado para llevar a cabo la reacción, es decir, se determinó estequiométricamente la cantidad de magnetita que se debería haber obtenido partiendo de las cantidades de reactivos agregados en la síntesis.

$$
\% \text { de rendimiento }=\frac{\text { Rendimiento real }}{\text { Rendimiento teórico }} \times 100
$$

$$
\text { Rendimiento teórico }=\frac{\text { Masa de magnetita }}{\text { Masa molecular de magnetita }}
$$

Usando las ecuaciones (5), (6) se determinan los respectivos rendimientos como se presenta en la Tabla 4.

A partir de los datos consignados en la Tabla 3 y en la Tabla 4 se observa que hay una mayor obtención de magnetita para el caso de M1 con respecto a M3; sin embargo, la muestra para la cual se obtuvo una mayor masa de magnetita fue M2 con un total de $2.273 \mathrm{~g}$ y a partir de ella un porcentaje de rendimiento
Tabla 4. Rendimientos de la reacción.

\begin{tabular}{|c|c|}
\hline Muestra & Rendimiento de la reacción $(\%)$ \\
\hline M1 & 14,99 \\
\hline M2 & 35,03 \\
\hline M3 & 7,53 \\
\hline
\end{tabular}

de $35.03 \%$, esto puede atribuirse al comportamiento de las reacciones descritas en las ecuaciones (1), (2) y (3), lo que se debe al hecho de que sea necesaria la formación de compuestos intermedios para que se dé el resultado esperado.

\section{Caracterización}

\section{Química}

\section{FTIR}

El FTIR se realizó para caracterizar las muestras de magnetita sintetizadas y para identificar interacciones de la superficie metálica con otros grupos funcionales. La Figura 4 muestra los espectros

Tabla 3. Masa de cáscara de papaya y de magnetita obtenida.

\begin{tabular}{|c|c|c|c|}
\hline Muestra sin reactivos & Masa de cáscara (g) & Muestra con reactivos & $\mathbf{M a s a}_{\mathbf{F e}} \mathbf{O}_{\mathbf{4}}(\mathbf{g})$ \\
\hline \multirow{2}{*}{ E1 } & \multirow{2}{*}{10.040} & $\mathrm{M} \mathrm{1}$ & 1.337 \\
\cline { 3 - 4 } & 10.054 & $\mathrm{M} 2$ & 2.273 \\
\hline E2 & $\mathrm{M} 3$ & 0.933 \\
\hline
\end{tabular}
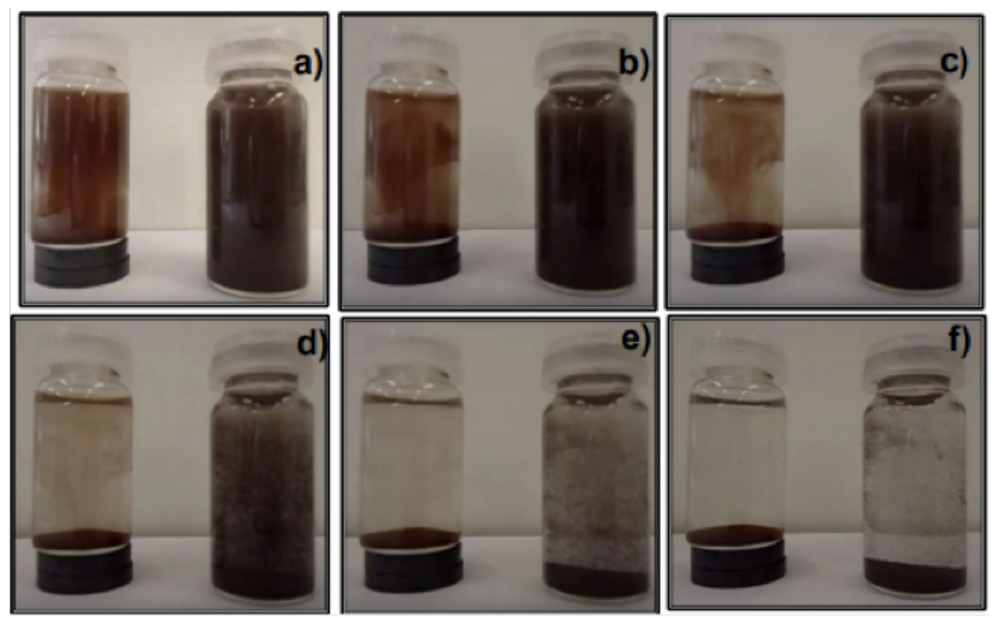

Figura 3. Fotografías de muestras de nanopartículas de magnetita en agua, evaluando magnetismo (frasco de la izquierda) frente a precipitación natural de las nanopartículas (frasco de la derecha). a) tiempo de colocación sobre el imán 0s, b) en un tiempo de $4 \mathrm{~s}$, c) $9 \mathrm{~s}$, d) $17 \mathrm{~s}$ e) $47 \mathrm{~s}$, f) $180 \mathrm{~s}$. 

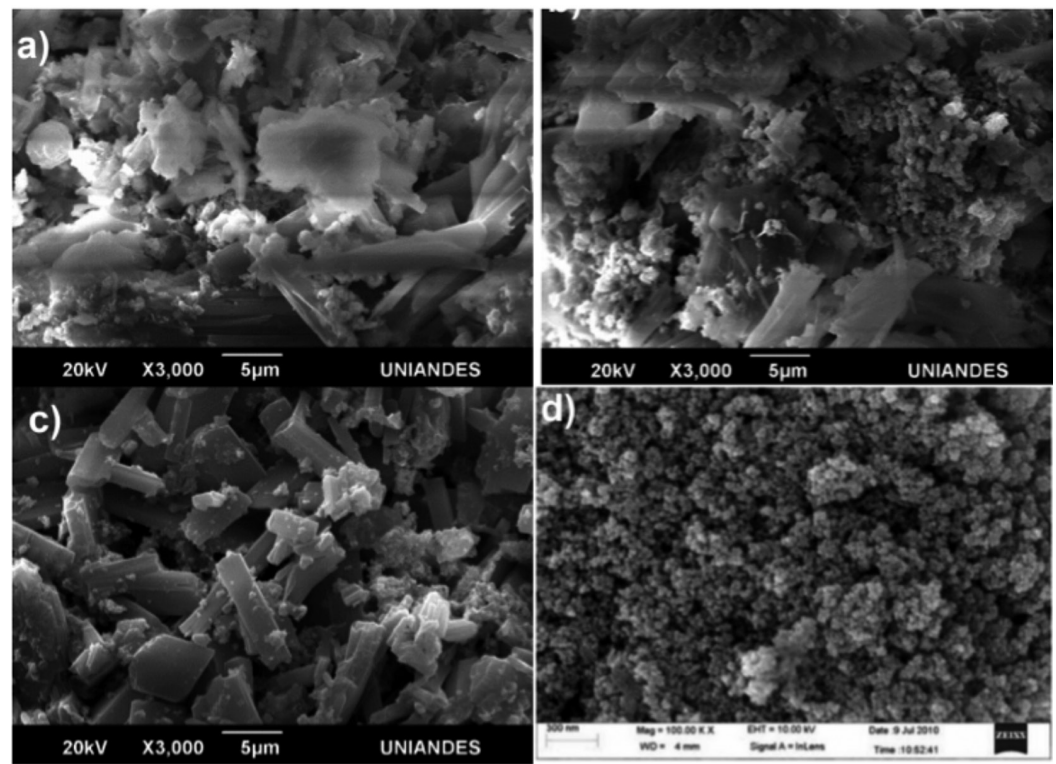

Figura 4. Micrografías SEM de los compuestos obtenidos. a) M1, b) M2, c) M3, d) Referencia magnificación 100000 x, voltaje $10 \mathrm{kV}$ (Solomon et al., 2012).

correspondientes a las tres muestras sintetizadas frente a una muestra comercial que comprenden picos similares, las bandas anchas en las longitudes 3261 y $3741 \mathrm{~cm}^{-1}$ de M1 y M2 respectivamente y al mostrar un pico más delgado en 1731 y $1732 \mathrm{~cm}^{-1}$ se puede decir que corresponden a vibraciones de flexión $\mathrm{H}-\mathrm{O}-\mathrm{H}$ mostrando que posiblemente las partículas de magnetita han fisisorbido agua en su superficie. Las bandas con longitudes de onda de 1447,1439 , y $1435 \mathrm{~cm}^{-1}$ para las muestras a, b, c, d, e, f corresponden a la vibración de deformación en enlaces O-H [24]. Por otro lado las bandas ubicadas en 2343,2338 y $2955 \mathrm{~cm}^{-1}$ equivaldrían a una tensión de tipo $\mathrm{C}-\mathrm{H}$, también es posible observar para las tres muestras un pico que se presenta en la zona alrededor de los 1000 y los $1200 \mathrm{~cm}^{-1}$ lo que indica alargamiento entre los enlaces $\mathrm{C}-\mathrm{O}$ [25], dicho comportamiento evidencia que aún hay presencia de compuestos secundarios formados por las reacciones presentadas en las ecuaciones (1), (2) y (3), por último los picos comprendidos entre 400 y $600 \mathrm{~cm}^{-1}$ se caracterizan por determinar la presencia de $\mathrm{Fe}-\mathrm{O}$ mostrándose como los picos 451 , $539 \mathrm{~cm}^{-1}$ para $\mathrm{M} 1 ; 472,560 \mathrm{~cm}^{-1}$ para M2 y 476 , $554 \mathrm{~cm}^{-1}$ para M3 evidenciando que hubo formación de óxidos de hierro [26], además de la presencia de picos en 839 y $864 \mathrm{~cm}^{-1}$ correspondiente a la presencia de Fe-OH para M2 y M3. Los espectros de las muestras de magnetita sintetizadas fueron comparadas frente al espectro de la magnetita como mineral comercial (óxido de hierro negro) arrojando los espectros superpuestos de la Figura 4, se observa que las muestras guardan varias similitudes con la magnetita comercial, entre esas, el pico entre $800 \mathrm{~cm}^{-1}$ y $400 \mathrm{~cm}^{-1}$ perteneciente a los enlaces tipo Fe-O que están ubicados dentro del mismo rango y una magnificación parecida, evidenciando que de la síntesis se obtuvo el compuesto esperado. Sin embargo, para el caso de la magnetita comercial se observa una región que abarca un ancho de banda desde $1800 \mathrm{~cm}^{-1}$ hasta $1000 \mathrm{~cm}^{-1}$, zona en la cual se pueden identificar diferentes grupos funcionales además de diferentes comportamientos entre los mismos, tales como compuestos aromáticos, en la zona entre $1680 \mathrm{~cm}^{-1}-1500 \mathrm{~cm}^{-1}$, deformación del enlace C-H en la zona entre $1475 \mathrm{~cm}^{-1}$ y $1300 \mathrm{~cm}^{-1}$, entre otros, por tal motivo no es posible identificar un solo tipo de enlace o interacción entre estos, indicando que existe la presencia de varios compuestos dentro de la magnetita comercial lo cual se puede atribuir al hecho de que es obtenida por minería, razón por la que esta se convierte en una matriz de varios compuestos tales como sílice, calcio entre otros [27] tal como se identifica 
en el FTIR, teniendo en cuenta que en general la procedencia natural influye en que haya presencia de más grupos además del grupo de interés para el producto deseado.

\section{Físico}

\section{Magnetismo}

En la Figura 3 se muestra la evaluación del magnetismo por parte de las nanopartículas comparada frente a la precipitación natural de éstas, el recipiente del lado izquierdo comprende un campo magnético en su base (inducido por un imán) mientras el del lado derecho de cada fotografía muestra la precipitación común de estas al mismo tiempo. Se puede evidenciar así que las nanopartículas llegan a la base en un tiempo de 17 segundos (Figura 3d) para la muestra que comprende un campo magnético, mientras que la otra muestra comprende una precipitación al cabo de 3 minutos, evidenciándose en la Figura 3f; aun así, no es una precipitación total puesto que aún se evidencian partículas por las paredes del vidrio.

\section{SEM}

Teniendo en cuenta que el fin de la presente investigación está relacionado con la obtención de nanopartículas de magnetita se procede a someter a microscopía electrónica de barrido las muestras resultantes, obteniendo como resultado las micrografías que se pueden observar en la Figura 4 (a, b y c).

Según se observa en la Figura 4 ( $a$, b y c), las muestras presentan dos tipos de morfología en ellas, esto puede deberse a que en la reacción (3) la relación molar entre los carbohidratos y la hematita necesarios para la formación de magnetita es de 1:3 lo que evidencia que la cantidad de carbohidratos añadida no reaccionó con la totalidad de la hematita formada. Por otro lado, se observa que la muestra con mayor porcentaje de magnetita (por características morfológicas) es la M2 (Figura 4b), en donde se puede evidenciar que se obtuvo magnetita nanoparticulada debido a la morfología agrupada muy similar a la reportada por literatura en la Figura 4d, además se puede determinar que las partículas de magnetita obtenidas tienen un tamaño nanométrico de aproximadamente $81.515 \mathrm{~nm}$ en promedio, dicha medida se determinó al hacer un aumento de escala (midiendo 5 partículas), teniendo en cuenta la escala de $5 \mu$ presentada en la micrografías.
Para el caso de M1 y M3 Figuras 4a y 4c respectivamente, predomina la morfología de bastones alargados y cuyo tamaño no es nanométrico; sin embargo, se pueden observar también las agrupaciones características de la magnetita aunque en una cantidad mucho menor con respecto a la que se puede ver en la fotografía correspondiente a M2 (Figura 4b), estos resultados permiten corroborar que el mayor rendimiento de magnetita obtenido es el que se da cuando el exceso de acetato de sodio con respecto al cloruro de hierro usado es 15: 1 molar.

\section{Remoción de colorantes}

Para evaluar las propiedades de degradación de las nanopartículas sintetizadas se tomó el colorante azul brillante $(\mathrm{AB})$ y se evaluó con las muestras M2 y M3, las cuales comprenden el exceso que mejor rendimiento obtuvo y el exceso reportado, respectivamente y así poder ser comparadas entre ellas, para ello se tomó un volumen de $5 \mathrm{~mL}$ de colorante con una concentración inicial de $0,075 \mathrm{mg} / \mathrm{L}$ para cada muestra evitando la agitación. La Figura 5 muestra cómo las nanopartículas de magnetita degradaron el colorante azul brillante. En el centro se encuentra la dilución de colorante como blanco, a ambos costados de esta se observan las dos muestras de magnetita mientras actúan removiendo el colorante, lo que se puede determinar cualitativamente al hacerse visible que el agua se tornó incolora.

\section{Espectroscopía UV-Vis en la remoción}

Las muestras fueron evaluadas por espectroscopía UV-Vis con método de barrido obteniendo los resultados de la Figura 6 donde la imagen $a$, corresponde a la dilución de colorante sin agregar magnetita, en este caso el agua con la dilución de

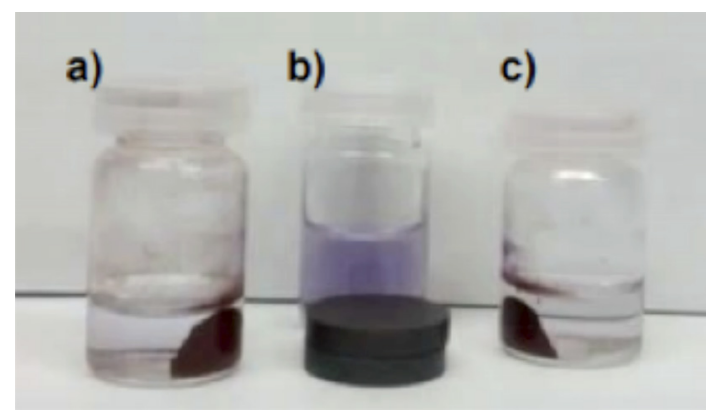

Figura 5. Degradación del colorante azul brillante. a) M2, b) blanco (muestra de colorante $\sin \mathrm{NPs}, \mathrm{c}) \mathrm{M} 3$. 


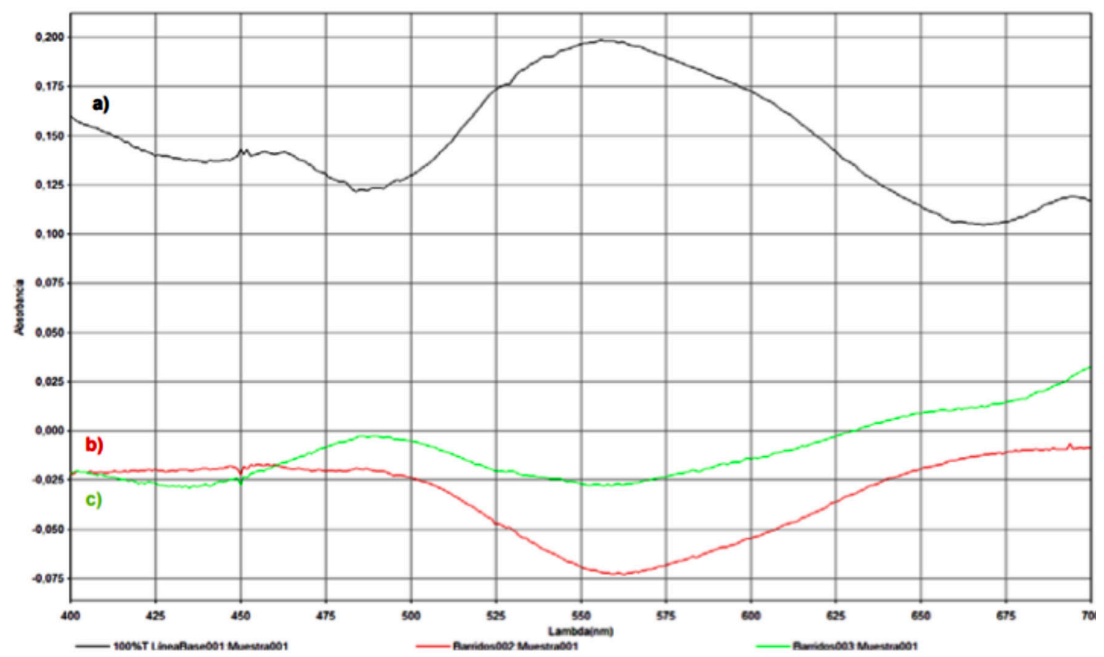

Figura 6. Espectroscopía UV-Vis método de barrido, a) muestra sin nanopartículas, b)M2, c) M3.

colorante, y la Figura $6 \mathrm{~b}$ y la Figura $6 \mathrm{c}$ pertenecientes a las muestras, se evidencia una menor absorción por parte de las muestras.

Por otro lado, con el fin de determinar cuánto tarda la magnetita obtenida en degradar el colorante $\mathrm{AB}$ se llevó a cabo el método fijo evaluando cómo disminuía la concentración de colorante al transcurrir el tiempo y cómo varía según la cantidad de magnetita agregada. Para este caso, a una dilución con colorante, se agregaron $0,1 \mathrm{~g}$ y $0,5 \mathrm{~g}$ aproximadamente, de las muestras 2 y 3 . Los resultados de la absorbancia se muestran en la Tabla 5.

Posteriormente, se realizó la curva de calibración respectiva del colorante $\mathrm{AB}$ Figura 7, para así

Tabla 5. Absorbancias determinadas en el tiempo.

\begin{tabular}{|c|c|c|c|c|}
\cline { 2 - 5 } \multicolumn{1}{c|}{} & \multicolumn{2}{c|}{ M2 } & \multicolumn{2}{c|}{ M3 } \\
\cline { 2 - 5 } \multicolumn{1}{c|}{} & $\mathbf{0 , 1 1 8} \mathbf{g}$ & $\mathbf{0 , 5 0 0} \mathbf{g}$ & $\mathbf{0 , 1 0 1} \mathbf{g}$ & $\mathbf{0 , 5 1 0} \mathbf{g}$ \\
\hline $\mathbf{t}$ (min) & \multicolumn{4}{c|}{ Absorbancias } \\
\hline 5 & 0,410 & 0,597 & 0,569 & 0,644 \\
\hline 20 & 0,371 & 0,544 & 0,435 & 0,531 \\
\hline 40 & 0,309 & 0,464 & 0,367 & 0,500 \\
\hline 60 & 0,246 & 0,333 & 0,255 & 0,426 \\
\hline 80 & 0,208 & 0,312 & 0,176 & 0,289 \\
\hline 100 & 0,199 & 0,248 & 0,185 & 0,259 \\
\hline
\end{tabular}

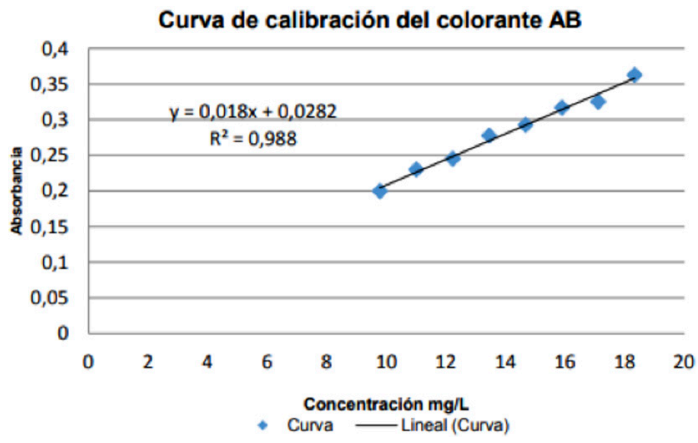

Figura 7. Curva de calibración correspondiente al colorante $\mathrm{AB}$.

determinar las concentraciones resultantes de la degradación. Por medio de la ecuación representada en la Figura 7 y las absorbancias presentadas en la Tabla 5, se lograron determinar las diferentes concentraciones presentadas durante el proceso de degradación Tabla 6.

Teniendo como punto de partida las concentraciones presentadas en la Tabla 6, se llevó a cabo el proceso de remoción verificando en el transcurrir del tiempo la disminución de la concentración de colorante como se ve en la Figura 8. Al comparar las diferentes cantidades de la magnetita resultante agregadas, se puede ver que actúa de manera más rápida la muestra M2 con respecto a la muestra M3, también es posible determinar que aumentar 
Tabla 6. Concentraciones determinadas con ayuda de la curva de calibración del colorante $\mathrm{AB}$ y las absorvancias.

\begin{tabular}{|c|c|c|c|c|}
\cline { 2 - 5 } \multicolumn{1}{c|}{} & \multicolumn{2}{c|}{ M2 } & \multicolumn{2}{c|}{ M3 } \\
\cline { 2 - 5 } \multicolumn{1}{c|}{} & $\mathbf{0 , 1 1 8} \mathbf{g}$ & $\mathbf{0 , 5 0 0} \mathbf{g}$ & $\mathbf{0 , 1 0 1} \mathbf{g}$ & $\mathbf{0 , 5 1 0} \mathbf{g}$ \\
\hline \multirow{2}{*}{ (min) } & \multicolumn{3}{c|}{ Concentraciones mg/L } \\
\hline 5 & 30,075 & 34,232 & 21,210 & 31,606 \\
\hline 20 & 22,638 & 27,954 & 19,048 & 28,658 \\
\hline 40 & 18,838 & 26,218 & 15,600 & 24,249 \\
\hline 60 & 12,605 & 22,094 & 12,136 & 16,928 \\
\hline 80 & 8,234 & 14,499 & 10,018 & 15,756 \\
\hline 100 & 8,712 & 12,819 & 9,494 & 12,236 \\
\hline
\end{tabular}

\section{Concentración vs Tiempo}

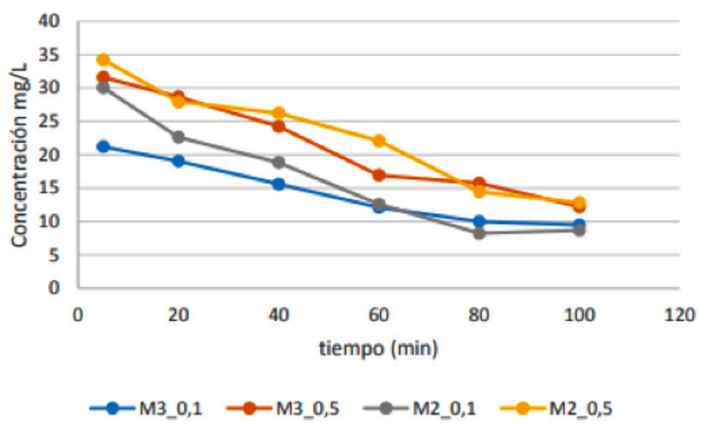

Figura 8. Concentración de colorante vs tiempo.

la cantidad de magnetita sí permitió que hubiese una mayor reducción de la cantidad de colorante presente en el agua, es importante resaltar que a pesar de ser una pequeña cantidad de magnetita agregada se logra una remoción importante en un corto periodo de tiempo (1h y $40 \mathrm{~min}$ ).

\section{CONCLUSIONES}

Se determinó que la cantidad de glucosa presente en la cáscara de papaya permite la obtención de nanopartículas de magnetita con el procedimiento descrito, esto se pudo comprobar por medio de la caracterización llevada a cabo por el método FTIR, en donde se evidencia el pico característico en una longitud de onda de $600-400 \mathrm{~cm}^{-1}$ perteneciente a enlaces del tipo $\mathrm{Fe}-\mathrm{O}$, pico característico de la magnetita comparada con una muestra comercial; sin embargo, no es posible atribuir la presencia de este pico solamente a la magnetita teniendo en cuenta que para su obtención es necesaria la formación de otro óxido de hierro (hematita), a pesar de ello se verificó que el compuesto resultante es magnético tal como lo es la magnetita debido a que se realizó una caracterización física con imanes. Por otro lado, gracias al porcentaje de rendimiento calculado se demostró que es posible disminuir el reactivo en exceso obteniéndose incluso una cantidad mayor de magnetita comparada con la cantidad obtenida cuando es agregado el mayor exceso estudiado.

Gracias al SEM se evidenció que el compuesto obtenido no es netamente magnetita, sino que existe la presencia (en algunos excesos más visibles) de otros compuestos; sin embargo, se observa que para uno de los excesos agregados la cantidad de magnetita obtenida es mayor, además de ser un material nanoparticulado con un tamaño de $81,515 \mathrm{~nm}$ aproximadamente.

Finalmente, a partir de la tendencia mostrada en la gráfica de concentración vs tiempo (Figura 8) se logra evidenciar la capacidad de degradación de la magnetita sintetizada, se debe tener en cuenta que la concentración de colorante usada fue baja haciendo que su degradación fuese más rápida.

\section{AGRADECIMIENTOS}

Las autoras expresan su agradecimiento al personal técnico y a la Universidad Jorge Tadeo Lozano, Laboratorio de Nanotecnología y al Semillero de Nanobioingeniería.

\section{REFERENCIAS}

[1] S. Abbaszadeh, H. Nodeh and S. Alwi. "Bio-adsorbent derived from papaya peel waste and magnetic nanoparticles fabricated for lead determination". Pure and Applied Chemistry. Vol. 90 No 1, pp. 83-90. 2017.

[2] J. Almaguer. "Contaminación de agua por colorantes y propuesta de remediación". Fecha de consulta: 01 de junio de 2018. URL: http://www.cucei.udg.mx/es/noticia/ contaminacion-de-agua-por-colorantes-ypropuesta-de-remediacion.

[3] S. Saval. "Aprovechamiento de residuos agroindustriales: pasado, presente y futuro". Sociedad Mexicana de Biotecnología y Bioingeniería. Vol. 16 Nº 1, pp. 14-46. 2012. 
[4] C.E. Quinilla Lindo. "Producción y comercialización de papaya híbrida Taynung carica papaya en la aldea San Francisco". Fecha de consulta: 16 de julio de 2011. 2017. URL: http://biblioteca.usac.edu.gt/ tesis/03/03_3896.pdf

[5] C. Mendes dos Santos, C.M. Patto de Abreu, J. Mesquita Freire, E. de Rezende Queiroz and M. Mendes Mendonça. "Chemical characterization of the flour of peel and seed from two papaya cultivars". Food Science and Technology (Campinas). Vol. $34 \mathrm{~N}^{\mathrm{o}} 2$, pp. 353-357. 2014.

[6] O.F. Castellanos, A.M. Fúquene M., S.L. Fonseca R., D.C. Ramírez, E. Giraldo T. y M.F. Valencia. "Estudio de la cadena productiva de la papaya en la región del norte del Valle (BRUT)”. pp. 8-10. 2011.

[7] D. Belandría, V. Velandría y C. Navarro. "Caracterización física, química y organoléptica de los frutos de lechosa (carica papaya $\mathrm{L}$ ) en las variedades Tailandia y Maradol". Producción Agropecuaria /Agroalimentaria. Vol. $3 \mathrm{~N}^{\circ}$ 1, pp. 45-49. 2010.

[8] S.M. Yepes, L.J. Montoya Naranjo y F. Orozco Sánchez. "Valorización de residuos agroindustriales-frutas en Medellín y el sur del Valle del Aburrá, Colombia". Revista Facultad Nacional de Agronomía. Medellín. Vol. $61 \mathrm{~N}^{\mathrm{o}}$ 1, pp. 4422-4431. 2008.

[9] A. Vanaamudan, H. Soni and P.P. Sudhakar. "Palm shell extract capped silver nanoparticles - As efficient catalysts for degradation of dyes and as SERS substrates". Journal of Molecular Liquids. Vol. $215 \mathrm{~N}^{\circ}$ 1, pp. 787-794. 2016.

[10] K.C. de Souza, G. Ferreira Andrade, I. Vasconcelos, V. de Oliveira, I.M. Fernandes and C.E.M. Barros. "Magnetic solid phase extraction based on mesoporous silica-coated magnetic nanoparticles for analysis of oral antidiabetic drugs and human plasma". Materials Science and Engineering C. Vol. 40 $\mathrm{N}^{\mathrm{o}}$ 1, pp. 275-280. 2014.

[11] R. Zanella. "Metodologías para la síntesis de nanopartículas: controlando forma y tamaño". Mundo Nano Revista Interdisciplinaria en Nanociencia y Nanotecnología. Vol. $5 \mathrm{~N}^{\mathrm{o}} 1$, pp. 69-81. 2012.

[12] C. Prasad, S. Gangadhara and P. Venkateswarlu. "Bio-inspired green synthesis of $\mathrm{Fe}_{3} \mathrm{O}_{4}$ magnetic nanoparticles using watermelon rinds and their catalytic activity". Applied Nanoscience. Vol. 6 N ${ }^{\circ}$ 6, pp.797-802. 2016.

[13] G. Picasso, J. Vega, R. Uzuriaga y G. Ruiz. "Preparación de nanopartículas de magnetita por los métodos sol-gel y precipitación: estudio de la composición química y estructura". Revista de la Sociedad Química del Perú. Vol. 7 N 3, pp. 170-181. 2012.

[14] S. Venkateswarlu, B. N. Kumar, B. Prathima, Y. SubbaRao and V. Jyothi. "A novel green synthesis of $\mathrm{Fe}_{3} \mathrm{O}_{4}$ magnetic nanorods using Punica Granatum rind extract and its application for removal of $\mathrm{Pb}$ (II) from aqueous environment". Arabian Journal of Chemistry. Vol. $12 \mathrm{~N}^{\circ}$ 4, pp. 588-596. 2014.

[15] S. Venkateswarlu, S. Rao, T. Balaji, B. Prathima and N. Jyothi. "Biogenic synthesis of $\mathrm{Fe}_{3} \mathrm{O}_{4}$ magnetic nanoparticles using plantain peel extract". Materials Letters. Vol. 100, pp. 241-244. 2013.

[16] R. Solomon, S. Lydia, J. Merlin and P. Venuvanalingam. "Enhanced photocatalytic degradation of azo dyes using nano $\mathrm{Fe}_{3} \mathrm{O}_{4}$ ". Journal of the Iranian Chemical Society. Vol. 9 N $^{\circ}$ 2, pp. 101-109. 2012.

[17] M Horst, V. Lassalle ans M. Ferreira. "Nanosized magnetite in low cost materials for remediation of water polluted with toxic metals, azo- and antraquinonic dyes". Frontiers of Environmental Science \& Engineering. Vol. $9 \mathrm{~N}^{\circ}$ 5, pp. 746-769. 2015.

[18] A.L. Rodríguez and H.R. Zea. "Modificación del proceso de reducción expansiva para la síntesis de nanopartículas de hierro". Scientiarum. Vol. $19 \mathrm{~N}^{\circ}$ 2, pp. 153-160. 2014.

[19] S. Venkateswarlu, B.N. Kumar, C.H. Prasad, P. Venkateswarlu and N. Jyothi. "Bio-inspired green synthesis of $\mathrm{Fe}_{3} \mathrm{O}_{4}$ spherical magnetic nanoparticles". Physica B: Condensed Matter. Vol. $449 \mathrm{~N}^{\mathrm{o}}$ 1, pp. 67-71. 2014.

[20] M. Amir, U. Kurtan and A. Baykal. "Synthesis and application of magnetically recyclable nanocatalyst $\mathrm{Fe}_{3} \mathrm{O}_{4} @ \mathrm{Nico} @ \mathrm{Cu}$ in the reduction of azodyes". Chinese Journal of Catalysis. Vol. 36 Nº $^{\circ}$, pp. 1280-1286. 2015.

[21] D.A Skoog, F.J. Holler and S.R. Crouch. "Aplicaciones de la espectrometría en el infrarrojo". Cengage Learning. Vol. 9, pp. 474. Porto Alegre, Brasil. 2009.

[22] X. Sun, C. Zheng, F. Zhang, Y. Yang, G. Wu, A. Yu and N. Guan. "Size 
Controlled Synthesis of Magnetite $\left(\mathrm{Fe}_{3} \mathrm{O}_{4}\right)$ Nanoparticles Coated with Glucose and Gluconic Acid from a Single Fe (III) Precursor by a Sucrose Bifunctional Hydrothermal Method". The journal of physical chemistry. Vol. $113 \mathrm{~N}^{\mathrm{o}} 36$, pp. 16002-16008.

[23] P.W. Atkins y L. Jones. Reactivos limitantes. En Principios de química. Los caminos del descubrimiento. pp. 88. Bogotá: Médica Panamericana. 2006.

[24] M. Puca, E.Tacuri, M. Hurtado, M. Guerrero, A. Figueroa, N. Rojas, J. Cjuno, S. García y R. López. "Estudio de las propiedades magnéticas de nanopartículas de poliestireno poroso cargadas con magnetita". Revista peruana de química e ingeniería química. Vol. $15 \mathrm{~N}^{\circ}$ 2, pp. 5-12. 2012.

[25] F.D. Amézequita López y D. Mendoza Olivares. Manual de prácticas para el curso de química Analítica. Interpretación de espectros de la región infrarroja. México. 2000.

[26] X. Sun, C. Zheng, F. Zhang, Y. Yang, G. $\mathrm{Wu}, \mathrm{A}$. Yu and N. Guan. "Size Controlled Synthesis of Magnetite $\left(\mathrm{Fe}_{3} \mathrm{O}_{4}\right)$ Nanoparticles Coated with Glucose and Gluconic Acid from a Single Fe (III) Precursor by a Sucrose Bifunctional Hydrothermal Method". The journal of physical chemistry. Vol. $113 \mathrm{~N}^{\circ} 36$, pp. 16002-16008.

[27] Secretaría de economía. "Perfil de mercado del Hierro-Acero". pp. 5-12. 2013. 